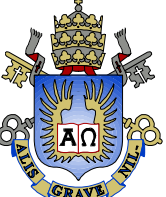

Alexandre Roberto Rentería

\title{
ESTIMAÇÃO DE PROBABILIDADE FUZZY A PARTIR DE DADOS IMPRECISOS
}

Tese apresentada ao Programa de Pós-graduação em Engenharia Elétrica da PUC-Rio como requisito parcial para obtenção do título de Doutor em Engenharia Elétrica

Orientador: Ricardo Tanscheit 


\section{Alexandre Roberto Rentería \\ Estimação de Probabilidade Fuzzy a partir de Dados Imprecisos}

Tese de Doutorado apresentada como requisito parcial para obtenção do grau de Doutor pelo Programa de Pós-Graduação em Engenharia Elétrica do Departamento de Engenharia Elétrica do Centro Técnico Científico da PUC-Rio. Aprovada pela Comissão Examinadora abaixo assinada.

Prof. Ricardo Tanscheit Orientador Departamento de Engenharia Elétrica - PUC-Rio

Profa. Marley Maria Bernardes Rebuzzi Vellasco Departamento de Engenharia Elétrica - PUC-Rio

Prof. Weiler Finamore Centro de Estudos em Telecomunicações - PUC-Rio

Prof. Juan Guillermo Lazo Lazo Departamento de Engenharia Elétrica - PUC-Rio

Prof. Alexandre Gonçalves Evsukoff UFRJ

Prof. Pedro Henrique Gouvêa Coelho UERJ

Prof. José Franco Machado do Amaral UERJ

Prof. José Eugenio Leal Coordenador Setorial do Centro

Técnico Científico - PUC-Rio 
Todos os direitos reservados. É proibida a reprodução total ou parcial do trabalho sem autorização da universidade, do autor e do orientador.

\section{Alexandre Roberto Rentería}

Graduou-se em Engenharia Elétrica, ênfase em eletrônica, pela PUC-Rio em 1999. Em 2002, obteve o título de mestre em Engenharia Elétrica em Métodos de Apoio à Decisão pela PUC-Rio. É membro do grupo de estudo em Inteligência Computacional do DEE/PUC-Rio. Vem realizando diversos projetos de pesquisa na área de automação industrial e instrumentação eletrônica desde 1999. Seus interesses incluem modelos matemáticos de incerteza e a aplicação da Teoria da Informação em sistemas inteligentes e em métodos de seleção de atributos.

Ficha Catalográfica

Rentería, Alexandre Roberto

Estimação de probabilidade fuzzy a partir de dados imprecisos / Alexandre Roberto Rentería ; orientador: Ricardo Tanscheit. - 2006.

94 f. : il. ; $30 \mathrm{~cm}$

Tese (Doutorado em Engenharia elétrica)-Pontifícia Universidade Católica do Rio de Janeiro, Rio de Janeiro, 2006.

Inclui bibliografia

1. Engenharia elétrica - Teses. 2. Probabilidade fuzzy. 3. Probabilidade imprecisa. 4. Janela de parzen. 5. Número fuzzy. 6. Incerteza de medição. 7. Estimação de probabilidade. 8. Estimação não-paramétrica. I. Tanscheit, Ricardo. II. Pontifícia Universidade Católica do Rio de Janeiro. Departamento de Engenharia elétrica. III Título. 


\section{Agradecimentos}

Ao meu orientador Professor Ricardo Tanscheit, sem cuja paciência e dedicação não teria sido possível realizar este trabalho.

Ao Professor Moisés Szwarcman, pelo apoio e compreensão.

À Professora Marley Vellasco, pelas importantes contribuições.

Aos meus pais e meus irmãos.

À minha amiga Babou, que me acompanhou durante a redação deste trabalho.

À PUC-Rio e à Vice-Reitoria Acadêmica, pelos auxílios concedidos. 


\section{Resumo}

Rentería, Alexandre Roberto; Tanscheit, Ricardo. Estimação de probabilidade fuzzy a partir de dados imprecisos. Rio de Janeiro, 2006. 94p. Tese de Doutorado Departamento de Engenharia Elétrica, Pontifícia Universidade Católica do Rio de Janeiro.

Existem três tipos de incerteza: a de natureza aleatória, a gerada pelo conhecimento incompleto e a que ocorre em função do conhecimento vago ou impreciso. Há casos em que dois tipos de incerteza estão presentes, em especial nos experimentos aleatórios a partir de dados imprecisos. Para modelar a aleatoriedade quando a distribuição de probabilidade que rege o experimento não é conhecida, deve-se utilizar um método de estimação nãoparamétrico, tal como a janela de Parzen. Já a incerteza de medição, presente em qualquer medida de uma grandeza física, dá origem a dados imprecisos, tradicionalmente modelados por conceitos probabilísticos. Entretanto, como a probabilidade se aplica à análise de eventos aleatórios, mas não captura a imprecisão no evento, esta incerteza pode ser melhor representada por um número fuzzy segundo a transformação probabilidade-possibilidade superior. Neste trabalho é proposto um método de estimação não-paramétrico baseado em janela de Parzen para estimação da probabilidade fuzzy a partir de dados imprecisos.

\section{Palavras-chave}

Probabilidade fuzzy; probabilidade imprecisa; probabilidade incerta; número fuzzy; incerteza de medição; estimação de probabilidade; estimação não-paramétrica; janela de Parzen 


\section{Abstract}

Rentería, Alexandre Roberto; Tanscheit, Ricardo. Fuzzy probability estimation from imprecise data. Rio de Janeiro, 2006. 94p. Doctoral Thesis - Departamento de Engenharia Elétrica, Pontifícia Universidade Católica do Rio de Janeiro.

There are three kinds of uncertainty: one due to randomness, another due to incomplete knowledge and a third one due to vague or imprecise knowledge. Sometimes two kinds of uncertainty occur at the same time, especially in random experiments based on imprecise data. To model randomness when the probability distribution related to an experiment is unknown, a non-parametric estimation method must be used, such as the Parzen window. Uncertainty in measurement originates imprecise data, traditionally modelled through probability concepts. However, as probability applies to random events but does not capture their imprecision, this sort of uncertainty is better represented by a fuzzy number, through the superior probability-possibility transformation. This thesis proposes a non-parametric estimation method based on Parzen window to estimate fuzzy probability from imprecise data.

\section{Keywords}

Fuzzy probability; imprecise probability; uncertain probability; fuzzy number; uncertainty in measurement; probability estimation; non-parametric estimation; Parzen window. 


\section{Sumário}

1 Introdução 13

1.1. Motivação 13

1.2. Objetivo 14

1.3. Estrutura da Tese 14

2 Incerteza $\quad 15$

2.1. Conceito geral de Incerteza 15

2.2. Incerteza como conceito mais amplo 16

2.2.1. O Paradoxo de Russell 16

2.2.2. Exemplo das Garrafas 17

2.2.3. Exemplo de Fang 20

2.3. Outros modelos de Incerteza 21

2.4. Incerteza de medição 21

2.4.1. Introdução 22

2.4.2. Definições 22

2.4.3. As causas das incertezas de medições e suas limitações 24

2.4.4. Medidas quantitativas da incerteza de medição 26

2.4.5. Avaliações do tipo A e do tipo B 28

2.4.6. Modelagem matemática 28

3 Teoria dos Conjuntos Fuzzy 30

3.1. Conceitos de Lógica Fuzzy 30

3.1.1. Conjuntos Fuzzy 30

3.1.2. Conjunto Singleton 32

3.1.3. Conjunto $\alpha$-cut 32

3.2. Conceitos de Números Fuzzy 33 
3.2.1. Intervalos

3.2.2. Número Fuzzy 34

3.2.3. Número Fuzzy Triangular 36

3.2.4. Aritmética de Intervalos 39

3.2.5. Aritmética Fuzzy 41

3.2.5.1. Operações do Intervalo $\alpha$-cut 41

3.2.5.2. Operações com o Número Fuzzy Triangular 42

4 Teoria da Probabilidade 48

4.1. Definição 48

4.2. Distribuição de probabilidade 49

4.2.1. A Distribuição Normal 49

4.2.2. Intervalo de Confiança 50

4.3. Métodos de Estimação de Probabilidade 50

4.4. Métodos de Estimação Não-Paramétricos 51

4.4.1. Definição 51

4.4.2. Histograma 52

4.4.3. Métodos de Kernel 54

4.4.4. Janela de Parzen 56

5 Modelo Proposto 59

5.1. Notação 59

5.2. Aritmética Fuzzy com Restrições Indispensáveis 60

5.3. Probabilidade Fuzzy 61

5.3.1. Caso Discreto 61

5.3.2. Caso Contínuo 68

5.3.3. Representação Gráfica $\quad 74$

5.4. Modelando a Incerteza de Medição Através de Números Fuzzy 77

5.5. Janela de Parzen Fuzzy 78

5.6. Exemplos 81

5.6.1. Exemplo 1: Amostras de uma Uniforme 81 
6 Conclusão

7 Referências Bibliográficas 


\section{Lista de figuras}

Figura 1 - Par de garrafas encontrado pelo homem no deserto 18

Figura 2 - Par de garrafas encontrado pelo homem no deserto (desvendadas) 19

Figura 3 - Gráfico da Probabilidade vs. Possibilidade 21

Figura 4 - Componentes de um Conjunto Fuzzy 31

Figura 5 - Exemplo de um Conjunto Singleton 32

Figura 6 - Exemplo de Conjunto $\alpha$-Cut 33

Figura 7 - Exemplo de Intervalo com $\mathrm{A}=\left[a_{1}, a_{3}\right]$

Figura 8 - llustração gráfica do número fuzzy $A=\left(a_{1}, a_{2}, a_{3}\right) \quad 35$

Figura 9 - $\alpha$-cut de um número fuzzy: $\alpha^{\prime}<\alpha \rightarrow A_{\alpha} \subset A_{\alpha^{\prime}}$

Figura 10 - Número fuzzy triangular $A=\left(a_{1}, a_{2}, a_{3}\right) \quad 37$

Figura 11 - Intervalo $\alpha=0,5$ cut do número fuzzy triangular $A=(-5,-1,1) \quad 39$

Figura $12-A(+) B$ e $A(-) B$ de números fuzzy triangulares $\quad 44$

Figura 13 - Multiplicação $A(\bullet) B$ de dois números fuzzy triangulares $\quad 46$

Figura 14 - Classificação dos métodos de estimação de densidade de probabilidade 51

Figura 15 - Dependência da forma do histograma em função da escolha da origem das células. $\quad 54$

Figura 16 - influência da largura da janela na estimativa por Janela de Parzen $\quad 58$

Figura 17 - Elementos de uma densidade de probabilidade uniforme fuzzy $\quad 70$

Figura 18 - Probabilidades fuzzy obtidas a partir de uma densidade uniforme fuzzy 72

Figura 19 - Representação gráfica de uma Normal de média nula e variância unitária, $f(x ; 0,1) \quad 75$

Figura 20 - Ilustração da desigualdade $\bar{P}[10,12]+\bar{P}[12,15] \supseteq \bar{P}[10,15]$ 
Figura 21 - Conjunto dos intervalos de confiança associados a uma distribuição normal

Figura 22 - Aproximação superior do conjunto de intervalos de confiança através de um número fuzzy triangular

Figura $23-\bar{P}[1,4]$ estimadas a partir de 100 amostras com incertezas de 0,5 e 1,0

Figura 24 - $\bar{P}[1,4]$ estimadas a partir de 1000 amostras com incertezas de 0,5 e 1,0

Figura $25-\bar{P}[10,15]$ estimadas a partir de 100 amostras com incertezas de 0,5 e 1,0

Figura $26-\bar{P}[10,15]$ estimadas a partir de 1000 amostras com incertezas de 0,5 e 1,0 


\section{Lista de tabelas}

Tabela 1 - Probabilidade vs. Possibilidade 20

Tabela 2 - $\alpha$-cuts das probabilidades fuzzy $\quad 76$

Tabela 3 - $\alpha$-cuts da estimativas de $\bar{P}[1,4]$ a partir de 100 amostras com incertezas de 0,5 e 1,0 82

Tabela 4 - $\alpha$-cuts da estimativas de $\bar{P}[1,4]$ a partir de 1000 amostras com incertezas de 0,5 e 1,0 84

Tabela 5 - Evolução da estimativa em função do número de amostras (exemplo 1) 85

Tabela 6 - $\alpha$-cuts da estimativas de $\bar{P}[10,15]$ a partir de 100 amostras com incertezas de 0,5 e 1,0

Tabela 7 - $\alpha$-cuts da estimativas de $\bar{P}[10,15]$ a partir de 1000 amostras com incertezas de 0,5 e 1,0

Tabela 8 - Evolução da estimativa em função do número de amostras (exemplo 2) 